Supporting information for

\title{
Tracing Single Electrons in a Disordered Polymer Film at Room Temperature
}

\author{
Kevin Wilma ${ }^{\mathrm{a}, \perp}$, Abey Issac ${ }^{\mathrm{a},{ }^{+}, \perp}$, Zhijian Chen $^{\mathrm{b}, \ddagger}$, \\ Frank Würthner ${ }^{b}$, Richard Hildner ${ }^{a}$, and Jürgen Köhler ${ }^{a,{ }^{*}}$
}

${ }^{a}$ Experimental Physics IV and Bayreuth Institute for Macromolecular Research (BIMF),

University of Bayreuth, 95440 Bayreuth, Germany

${ }^{b}$ Institut für Organische Chemie and Center for Nanosystems Chemistry, Universität Würzburg, Am Hubland, 97074 Würzburg, Germany

*Corresponding authors: juergen.koehler@uni-bayreuth.de

$\perp$ these authors contributed equally to this work

${ }^{\dagger}$ Current address:

Department of Physics, Sultan Qaboos University 123 Muscat (Oman)

\section{${ }^{\ddagger}$ Current address:}

School of Chemical Engineering and Technology, and Collaborative Innovation Center of Chemical Science and Chemical Engineering (Tianjin)

Tianjin University

300072 Tianjin (China)

\section{Contents}

1. Methods

2. Simultaneous measurements of spectra and lifetimes of single PBI 1 molecules 4

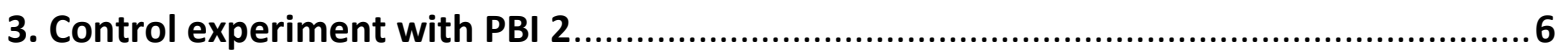

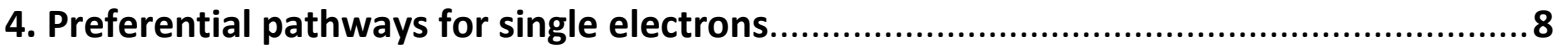

5. Nature and origin of charge carriers captured by single PBI molecules ......................10

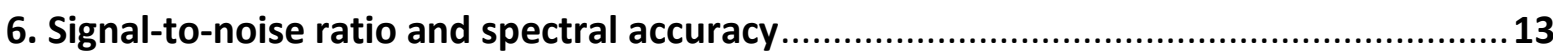




\section{Methods}

We used two perylene bisimide derivatives as probe molecules, i.e. $N, N^{\prime}$-Di(2,6-di-isopropylphenyl)-1,6,7,12-tetra(4-tert-butylphenoxy)perylene-3,4:9,10-tetracarboxylic acid bisimide (PBI 1) and N,N'-Di-(3,4,5-tridodecylphenyl)-perylene-3,4:9,10-tetracarboxylic acid bisimide (PBI 2), see Fig. S.1 which were synthesized according to literature known procedures [1].

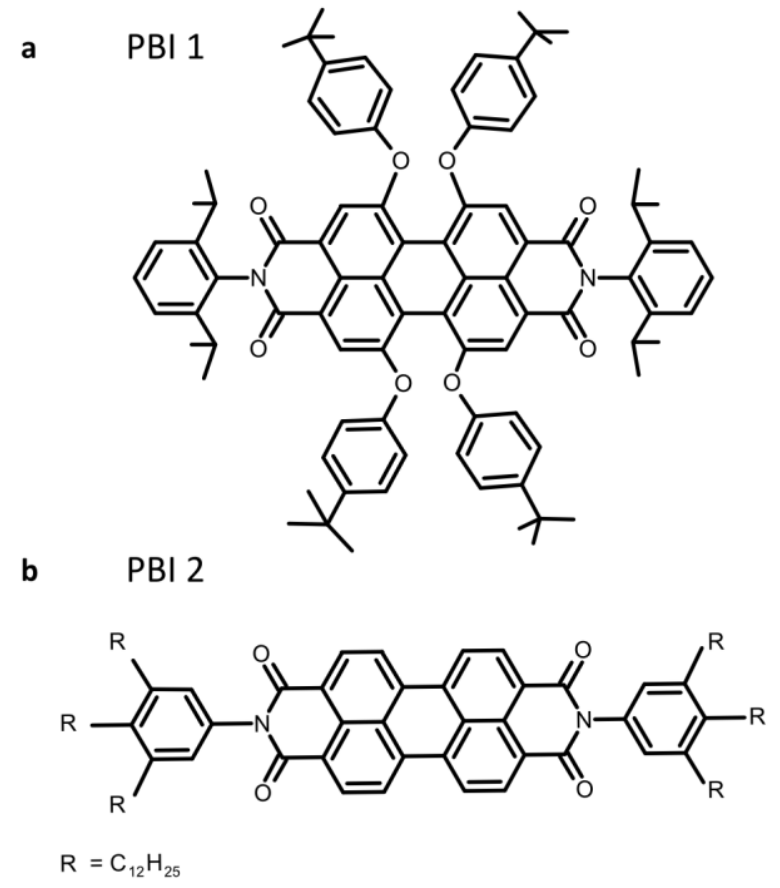

Fig. S.1. Schematic sketch of the structure of the two PBI derivatives used in this study.

For ensemble experiments the chromophores were dissolved at a concentration of $10^{-6} \mathrm{M}$ in toluene (Sigma-Aldrich, 99.7\%). Steady-state absorption and emission spectra were registered using commercial spectrometers (absorption: Perkin-Elmer Lamda-750; emission: Varian Cary Eclipse). The emission spectra were excited at $532 \mathrm{~nm}$. For the single-molecule experiments the $\mathrm{PBI}$ molecules were dissolved at about $10^{-10} \mathrm{M}$ in toluene that contained $5 \mathrm{mg} / \mathrm{ml}$ polystyrene. Subsequently, 30 ? of this solution were spin-coated for $60 \mathrm{~s}$ on a freshly cleaned glass cover slip at 2500 revolutions per minute, which resulted in thin polymer films with a thickness of some 100 $\mathrm{nm}$.

The individual PBI molecules were excited with a pulsed diode laser (LDH-P-FA-530 L, PicoQuant) operating at $532 \mathrm{~nm}$ with a repetition rate of $20 \mathrm{MHz}$ (pulse width (FWHM) < $100 \mathrm{ps)}$. The sample was excited through a home-built confocal microscope using a dichroic beam splitter 
(z532RDC, AHF Analysentechnik AG) and an infinity-corrected oil-immersion objective (PlanApo, $60 x, N A=1.45$, Olympus). The excitation intensity in the focal plane was $4 \mathrm{~kW} / \mathrm{cm}^{2}$. Individual molecules could be positioned within the confocal volume employing a piezo stage (Tritor 102 SG, Piezosystem Jena). Their emission was collected by the same objective and passed through the dichroic beam splitter and a dielectric filter (545 LP, AHF Analysentechnik AG) that suppressed scattered excitation light. For the spectrally resolved measurements the signal was dispersed in a spectrometer (SPEC 250IS, 150 grooves/mm, $500 \mathrm{~nm}$ blaze, Bruker Optics Inc.) and recorded with a back-illuminated electron-multiplying charge-coupled device camera (emCCD, iXon DV887-BI, Andor Technology). The spectral resolution of the system was $30 \mathrm{~cm}^{-1}(1 \mathrm{~nm}$ at $600 \mathrm{~nm}$, see SI section 6). From each molecule we recorded typically 2000 emission spectra with an exposure time of $30 \mathrm{~ms}$. The resulting spectra were fitted with a combination of two Gaussian functions, one for the purely electronic transition $(0-0 ; Z P L)$ and the second for the vibrational side band. This approach yields simultaneously the energy of the peak of the emission spectrum $\bar{v}_{\text {peak }}$, and the total fluorescence intensity as a function of time. The latter is obtained by spectrally integrating the intensity of the individual spectra.

In order to detect simultaneously the fluorescence lifetime and the spectrum of a single PBI molecule we included a non-polarizing beam splitter cube into the detection beam path, directing $70 \%$ of the fluorescence signal to the spectrometer and $30 \%$ to a single photon counting photodiode (PDM Series, PicoQuant). Lifetime decays were accumulated using a time-correlated single-photon counting (TCSPC) module (TimeHarp 200, PicoQuant). Each fluorescence decay curve was integrated for $100 \mathrm{~ms}$ with a temporal resolution of $200 \mathrm{ps}$.

Sample preparation and all experiments were performed under ambient conditions. 


\section{Simultaneous measurements of spectra and lifetimes of single PBI 1 molecules}

a)

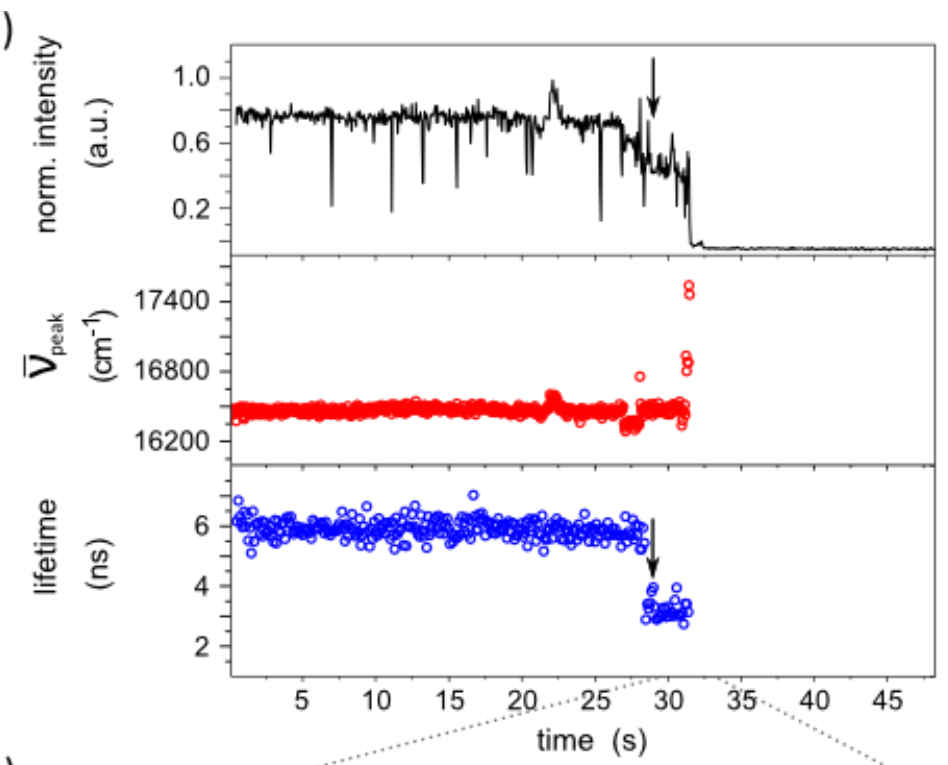

b)

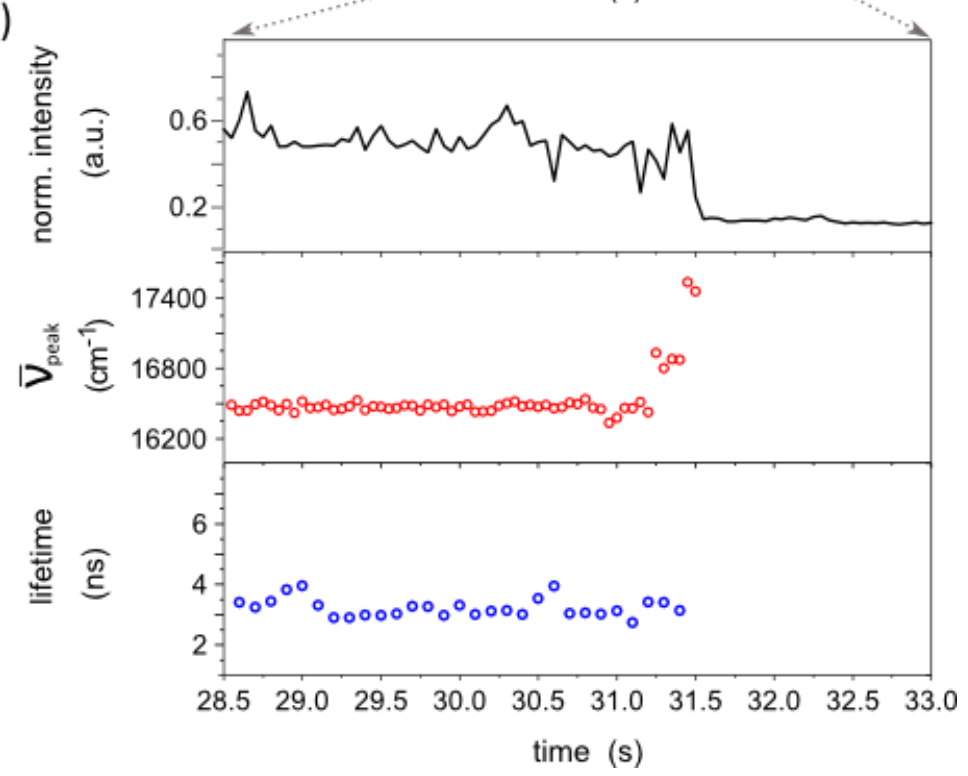

FIG. S.2. Example of a single PBI 1 molecule, for which the total fluorescence signal, the spectral peak position, and the lifetime was recorded simultaneously as a function of time. a) Top: Total fluorescence intensity; centre: energy of the peak of the emission spectrum; bottom: lifetime. b) Expanded view of the time interval from 28.5 to $33 \mathrm{~s}$ of the data presented in a.

Figure S.2 shows an example of a simultaneous measurement of spectra and excited state lifetimes from a single PBI 1 molecule (note that this is the same molecule as shown in Fig. 2a, b of the main text, where the lifetime trajectory was omitted for clarity). The trajectory of the total fluorescence intensity (Fig. S.2a, top) is terminated after $31 \mathrm{~s}$ by an irreversible photobleaching 
event caused by the formation of a charged photoproduct after capture of an electron from the PS matrix [2], which results in a blue shift of the spectral peak position by about $1000 \mathrm{~cm}-1$ (Fig. S.2 a, centre). The corresponding lifetime trajectory (Fig. S.2a, bottom) shows a constant lifetime of about 6 ns during the first $27 \mathrm{~s}$, which is characteristic for PBI molecules. For the remaining $4 \mathrm{~s}$ of the trajectory the lifetime drops to a constant value of $\sim 3.5 \mathrm{~ns}$. Since this decrease in lifetime is accompanied by a decrease in the total fluorescence intensity (Fig. S.2a, arrow), we attribute it to a matrix-induced change of the PL quantum efficiency $[2,3]$.

An expanded view of the trajectories from 28.5 to $33 \mathrm{~s}$ is displayed in Fig. S2 b, which clearly demonstrates that, although there is a strong, continuous blue shift of the peak emission wavelength, the excited state lifetime does not change during this time interval. This observation shows that neither the twist angle between the naphthalene units of PBI [4] nor the arrangement of the PS matrix around the PBI molecule changes substantially [5], which both give rise to (correlated) variations of lifetimes and spectra. The spectral shift observed here can therefore be associated exclusively with a Stark shift induced by the approach of an electron towards the PBI molecule. 


\section{Control experiment with PBI 2}

In order to test, whether conformational changes of the phenoxy-groups at the bay positions of $\mathrm{PBI} 1$ can give rise to the observed spectral shifts immediately before a blinking event, we performed similar experiments on a PBI derivative that lacks the phenoxy-groups. This derivative is referred to as PBI 2, see Fig S.1.

In Fig. S.3 (a) we show the recorded time trace of the total fluorescence intensity (top) and the peak position of the emission spectrum (bottom) from a PBI 2 molecule. Part (b) of the figure displays an expanded view from $39.7 s-41.1 \mathrm{~s}$ of the same trajectory. Between $40.4 \mathrm{~s}-40.8 \mathrm{~s}$ the intensity features a jump from the on- level to the background. Correlated with this blinking event is a spectral blue-shift of about $124 \mathrm{~cm}^{-1}$ (see Table S.1).
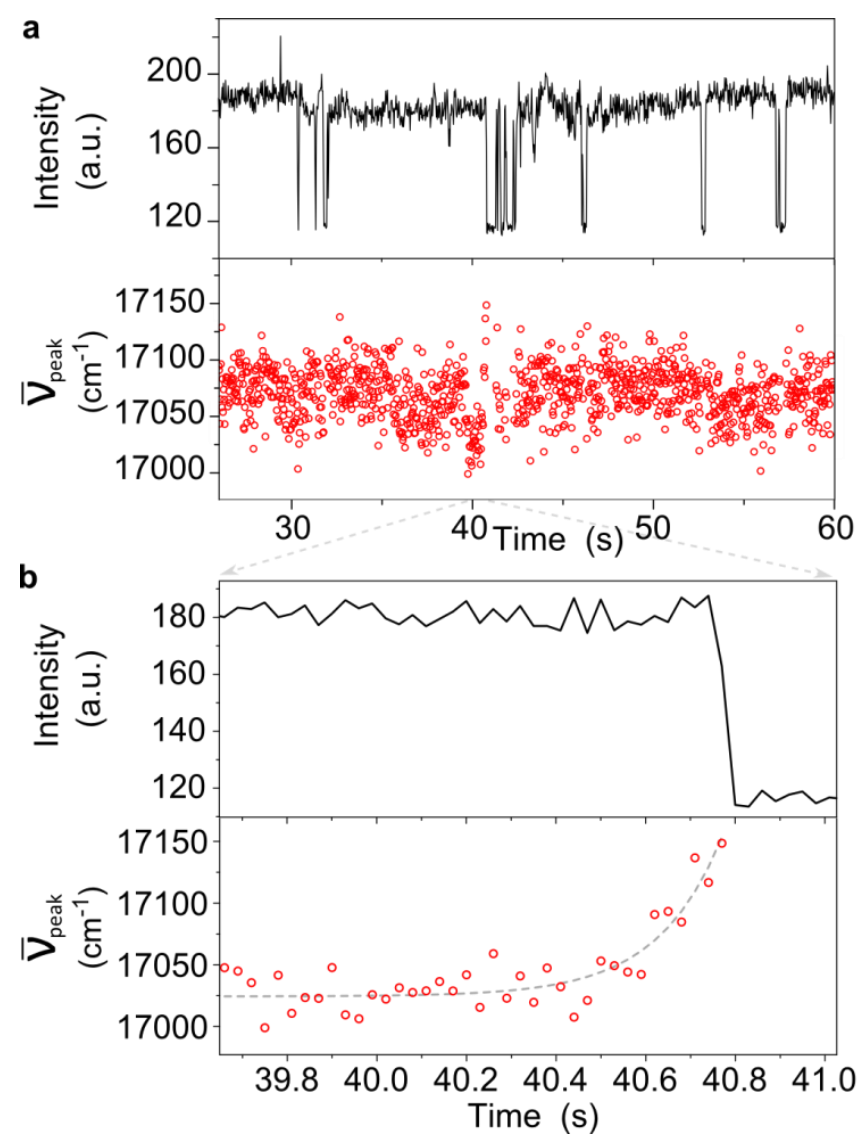

FIG. S.3. (a) Top: Fluorescence intensity (black line) of a single PBI 2 molecule as a function of time. Bottom: Simultaneously recorded energy of the emission peak (red circles). (b) Expanded view of the time interval $39.7 \mathrm{~s}-41.1 \mathrm{~s}$ of the data presented in (a). The parameters of the fit (dashed grey line) are listed in Table S.1. 


\begin{tabular}{l|l} 
parameter & blue shift / capture \\
\hline $\bar{v}_{0}$ & $(17024 \pm 15) \mathrm{cm}^{-1}$ \\
$\bar{v}_{\mathrm{A}}$ & $(238 \pm 15) \mathrm{cm}^{-1}$ \\
\cline { 2 - 2 }$\tau$ & $(0.143 \pm 0.025) \mathrm{s}$ \\
\cline { 2 - 2 }$t_{0}$ & $(40.7 \pm 0.025) \mathrm{s}$ \\
$\Delta \bar{v}_{\max }$ & $(124 \pm 15) \mathrm{cm}^{-1}$ \\
\cline { 2 - 2 }$\Delta \bar{v}_{1 / e}$ & $(88 \pm 15) \mathrm{cm}^{-1}$ \\
\cline { 2 - 2 }$r_{\min }$ & $(1.19 \pm 0.15) \mathrm{nm}$
\end{tabular}

TAB. S.1. Fit parameters of PBI 2 (Fig. S.3). Accuracies reflect the experimental resolution. 


\section{Preferential pathways for electron migration}

A further example that shows correlation between blinking and spectral shift is provided in Fig. S.4 (a). It displays the total fluorescence intensity (top) and spectral peak position $\bar{v}_{\text {peak }}$ (bottom) as a function of time for a PBI 1 molecule.
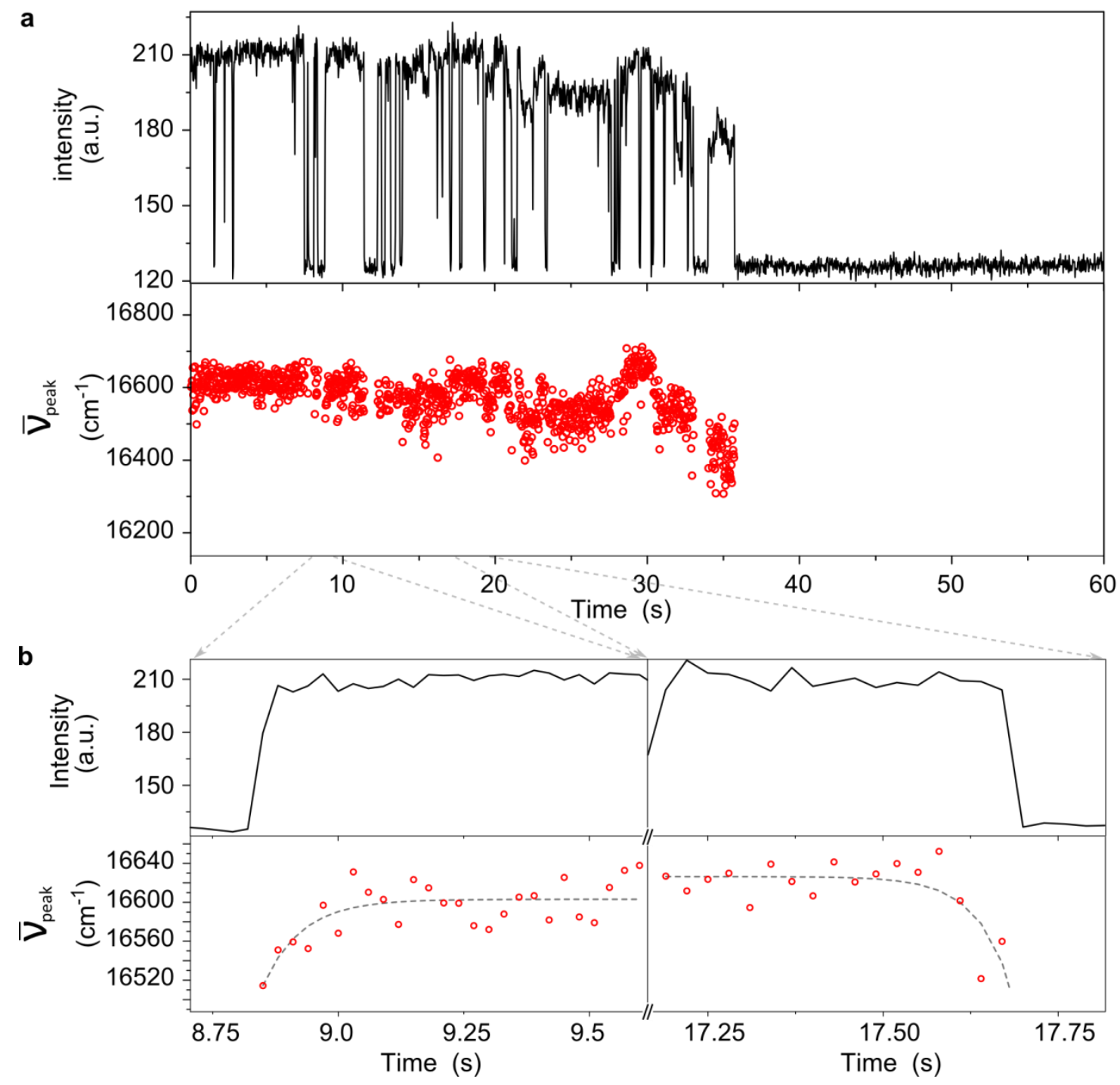

FIG. S.4. (a) Top: Fluorescence intensity (black line) of a single PBI 1 molecule as a function of time. Bottom: Simultaneously recorded spectral position of the emission peak (red circles). (b) Left (right) panel: Expanded view of the time interval $8.7 \mathrm{~s}-9.6 \mathrm{~s}(17.1-17.8 \mathrm{~s})$ of the data presented in (a). The optimized parameters of the fits (dashed grey lines) are listed in Table S.2. 
The respective events around $9 \mathrm{~s}$ and $17.5 \mathrm{~s}$ are shown in Fig. S.3 (b) on expanded scales. The parameters extracted from these data are summarized in Table S.2. The striking observation is that the values found for the release of an electron (bottom left) and those found for the capture of (probably another) electron (bottom right) some seconds later agree within experimental accuracy. This similarity is a strong indication that the electrons follow specific pathways within a particular part of the host matrix.

\begin{tabular}{l|l|l} 
parameter & blue shift / release & red shift / capture \\
\hline$\tau$ & $(0.077 \pm 0.025) \mathrm{s}$ & $(0.049 \pm 0.025) \mathrm{s}$ \\
$\Delta \bar{\nu}_{\max }$ & $(89 \pm 15) \mathrm{cm}^{-1}$ & $(105 \pm 15) \mathrm{cm}^{-1}$ \\
$\Delta \bar{\nu}_{1 / e}$ & $(25 \pm 15) \mathrm{cm}^{-1}$ & $(25 \pm 15) \mathrm{cm}^{-1}$ \\
$r_{\min }$ & $(1.81 \pm 0.15) \mathrm{nm}$ & $(1.57 \pm 0.15) \mathrm{nm}$
\end{tabular}

TAB. S.2. Fit parameters extracted from the data shown in Fig. S.4. Accuracies reflect the experimental resolution. 


\section{Nature and origin of charge carriers captured by single PBI molecules}

The spectral shifts observed for single PBI molecules immediately before a blinking event are ascribed to the approach of single charge carriers. For the following reason we associate these charge carriers with electrons (and not with holes):

First, in the language of solid state physics it is common to distinguish between electrons and holes. Owing to the large number of electrons $\left(10^{20}\right)$ it is much more convenient to consider the electron that is missing, i.e. the hole, rather than $10^{20}-1$ electrons. Yet, the physical mechanism of hole transport through a crystal in one direction is nothing else than the motion of electrons in exactly the opposite direction. In contrast, in an organic molecule the number of electrons is substantially smaller with respect to a solid (some 100), and thus the concept of a hole does not make much sense. This holds true in particular for the current situation where we discuss the migration of a single charge carrier. The molecular properties of $\mathrm{PBI}$ are substantially altered upon electron transfer either from PBI to PS or from PS to PBI. In our experiment the former situation (electron transfer from $\mathrm{PBI}$ to $\mathrm{PS}$, or in solid state language hole transfer from $\mathrm{PS}$ to $\mathrm{PBI}$ ) is not observable, because once the electron has left the PBI molecule a PBI cation remains which features absorption and emission spectra that are strongly shifted with respect to the neutral species. As a consequence of this, the PBI cation would be no longer resonant with our excitation light and photoluminescence will be ceased. Yet, the latter situation, i.e. an electron from the PS matrix that approaches the neutral PBI can be clearly detected by the influence of its electric field on the electronic transition energy of the chromophore.

Second, in a series of papers Fabish and Duke measured the density of states (DOS) for the anions in polystyrene films by injecting charges into the material $[6,7]$. This DOS is compared with the HOMO and LUMO levels of PBI 1 and PBI $2,{ }^{1}$ that have been calculated from the redox potentials measured in cyclic voltammetry and the energy gap determined from the optical spectra [8], see Fig. S.5. We find a substantial overlap between the DOS for the anionic species in PS and the energy of the LUMO of both PBI derivatives (ca. $-3.65 \mathrm{eV}$ for PBI 1 and ca. $-3.79 \mathrm{eV}$ for PBI 2, both numbers are relative to the vacuum level). Hence, highly reversible capture and release of an

\footnotetext{
${ }^{1}$ We note that HOMO and LUMO are theoretical constructs only and represent the one-electron molecular orbitals of the neutral PBI molecule. The HOMO and LUMO energies are commonly associated with the potential for formation of the cation and anion, respectively. However, these energies cannot be measured experimentally, only the energy difference between the electronic ground state and first excited singlet state is experimentally accessible.
} 
electron by the PBI molecule from/to the PS matrix is likely. In contrast, the HOMO levels (ca. $5.78 \mathrm{eV}$ for $\mathrm{PBI} 1$ and $-6.14 \mathrm{eV}$ for $\mathrm{PBI} 2$ ) are close to the minimum of the density of charged species in PS. This implies that the formation of a PBI cation by transferring an electron from the HOMO level to the PS matrix is very unlikely.

Although there is no unambiguous evidence, it is highly likely that electron transfer from the polystyrene host to the embedded PBI molecules occurs and gives rise to the reversible blinking events observed.

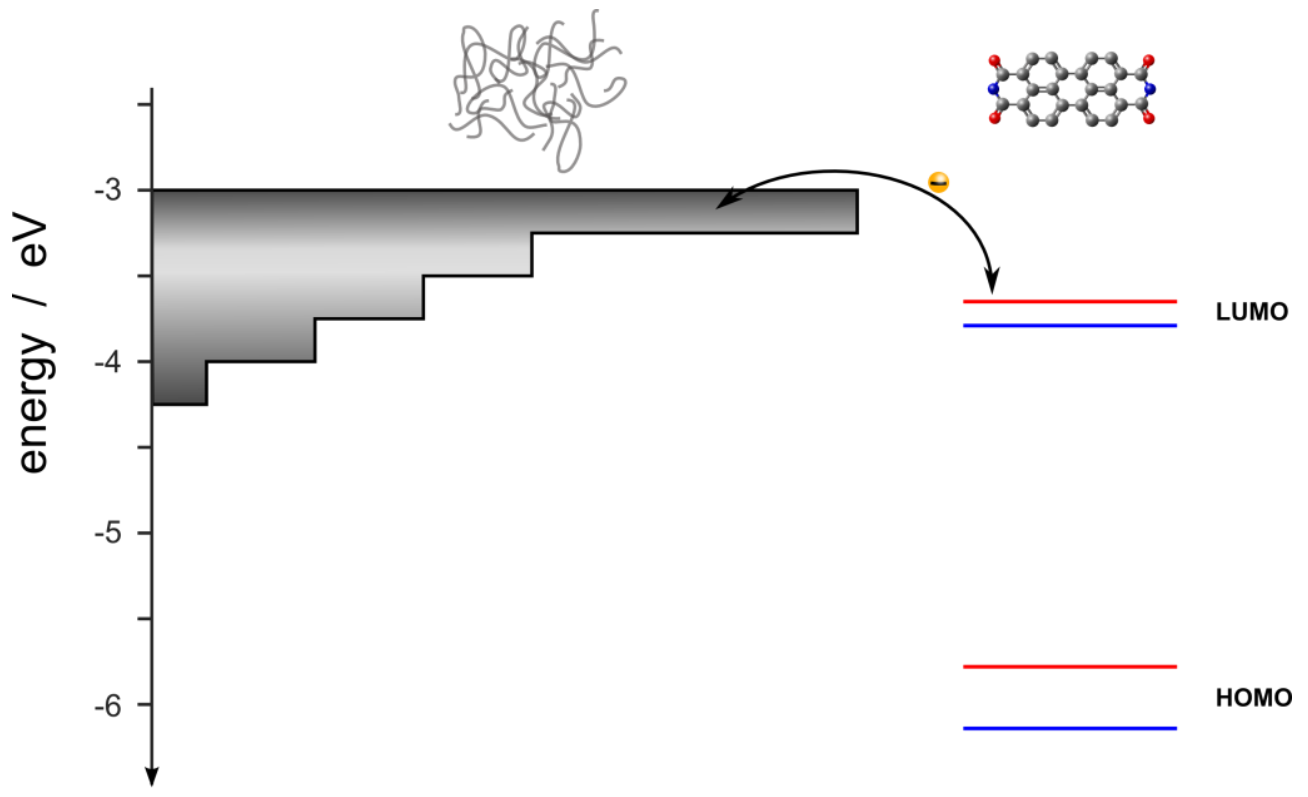

FIG. S.5. Experimentally determined anion density of states (grey shaded area) of a polystyrene film [6], and the calculated LUMO and HOMO levels of PBI 1 (red solid lines) and PBI 2 (blue solid lines). All levels are given relative to the vacuum level.

The origin of these "free" electrons in polystyrene is probably related to the synthesis and purification process. Polystyrene is synthesized via radical polymerisation, i.e. it is formed by successive addition of free radical building blocks. The synthesis involves suitable initiator molecules that are prone to radical formation. We therefore suggest that the free electrons that give rise to blinking of $\mathrm{PBI}$ originate from such radicals created during synthesis, because a perfect purification (down to the single electron / single molecule level) is certainly not possible.

Finally, we provide an estimate of the density of "free" electrons in the PS film: The volume of our samples is in the order of $10^{-11} \mathrm{~m}^{3}(1 \mathrm{~cm} \times 1 \mathrm{~cm} \times 100 \mathrm{~nm})$. In the single-molecule experiments the concentration of $\mathrm{PBI}$ is typically $10^{-10} \mathrm{M}$ corresponding to $10^{-10} \mathrm{PBI}$ molecules $/ \mathrm{nm}^{3}$. If we further assume that one electron is present per PBI molecule, we obtain about $10^{-10}$ electrons $/ \mathrm{nm}^{3}$ or equivalently $10^{11}$ electrons $/ \mathrm{cm}^{3}$, which is clearly a very low density of charge carriers characteristic 
for an electrical insulator. Even if there were 10000 electrons per single PBI molecule, the resulting charge carrier density would still be that of an insulator. 


\section{Signal-to-noise ratio and spectral accuracy}

As mentioned in the main text, each individual emission spectrum in a sequence of spectra from a single molecule was fitted by the sum of two Gaussian functions, see Fig. S.6.

A quantitative estimate for the accuracy with which we can determine the spectral peak position $\bar{v}_{\text {peak }}$ of the emission maximum from single molecule spectra and as a consequence of this the precise value of the spectral shifts observed before and after the blinking events is then obtained from the error of the fit. For the purely electronic transition (ZPL) of a single molecule emission spectrum this yields an accuracy of about $10 \mathrm{~cm}^{-1}(0.4 \mathrm{~nm}$ at $600 \mathrm{~nm})$.

As an alternative approach to estimate the accuracy for the spectral peak position we calculated the signal-to-noise ratio (SNR). From our data we obtain a typical SNR of ca. 20. Based on the number of the detected photons (see. Fig S.6) we expect a shot noise limited SNR of ca. 40, indicating that laser power fluctuations contribute as well, whereas detector noise is efficiently suppressed because during the measurements the camera was cooled to $-80{ }^{\circ} \mathrm{C}$. Sbalzarini et al. have shown that the accuracy of determining a distinct position on a pixelated device is better than 0.1 pixel if the SNR reaches a value of 4.2 [9]. Our SNR, however, is substantially bigger than this critical value, allowing us a very accurate spectral position determination of significantly better than 1 pixel on the CCD camera, i.e. better than $30 \mathrm{~cm}^{-1}(1 \mathrm{~nm}$ at $600 \mathrm{~nm})$.

Although both methods yield similar values clearly below one pixel or $30 \mathrm{~cm}^{-1}$, we conservatively assume $30 \mathrm{~cm}^{-1}$ as the accuracy of the spectral peak positions. 


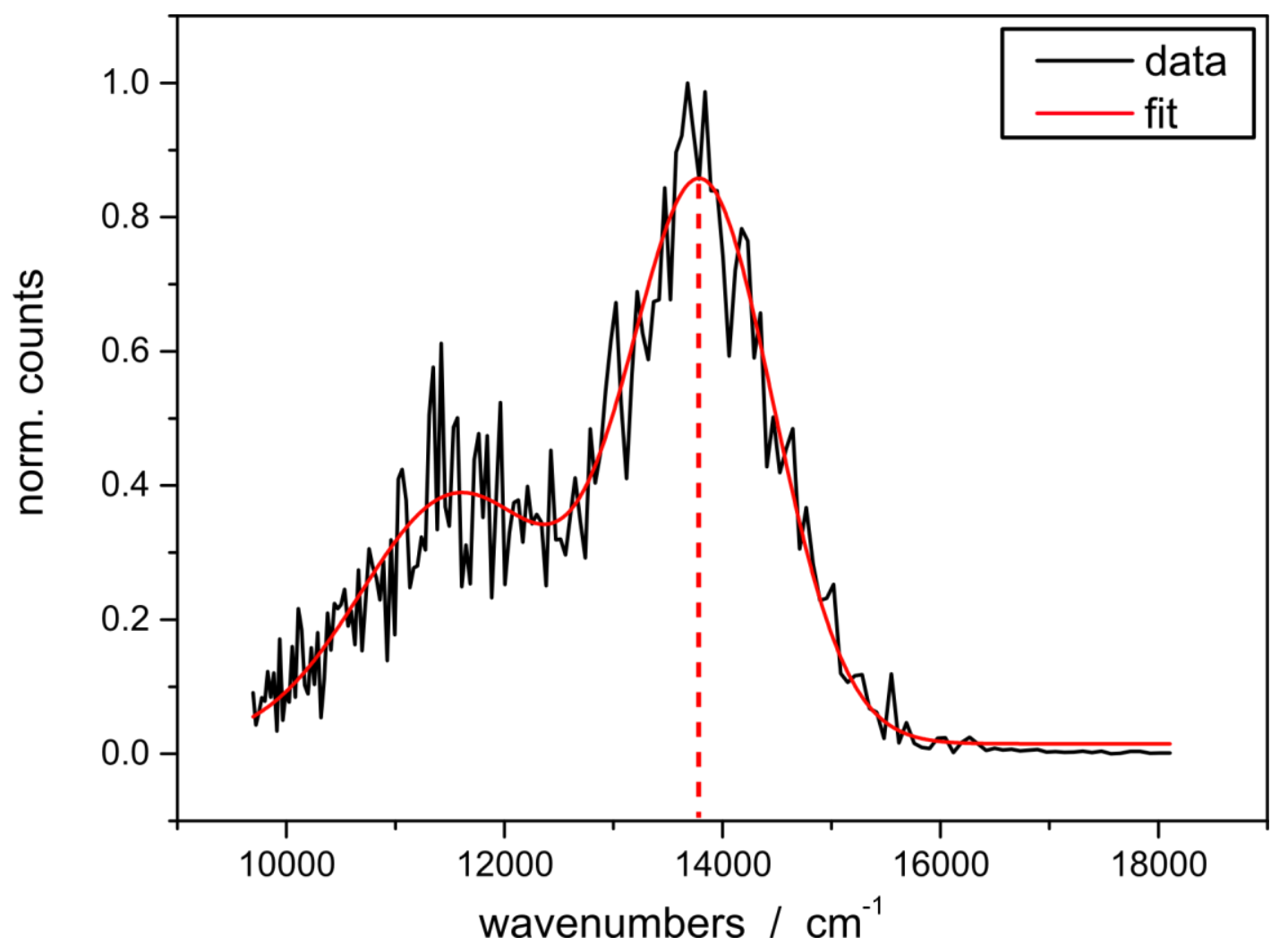

FIG. S.6. Emission spectrum of a single PBI 1 molecule taken with an exposure time of 50 ms (black solid line). The red curve is a fit to the data with a sum of two Gaussian functions. The vertical dashed line indicates the spectral peak position of the purely electronic transition. 
[1] Chen, Z.; Stepanenko V.; Dehm V.; Prins , P.; Siebbeles, L.D.; Seibt, J.; Marquetand, P.; Engel, V.; Würthner F.; Photoluminescence and Conductivity of Self-Assembled Pi-Pi Stacks of Perylene Bisimide Dyes. Chem. Eur. J. 2007, 13, 436 - 49.

[2] Issac, A.; Hildner, R.; Ernst, D.; Hippius, C.; Würthner, F.; Köhler, J. Single Molecule Studies of Calix[4]arene-Linked Perylene Bisimide Dimers: Relationship between Blinking, Lifetime and/or Spectral Fluctuations. Phys. Chem. Chem. Phys. 2012, 14, $10789-10798$.

[3] Issac, A.; Hildner, R.; Hippius, C.; Würthner, F.; Köhler, J. Stepwise Decrease of Fluorescence versus Sequential Photobleaching in a Single Multichromophoric System. Am. Chem. Soc. Nano 2014, 8, 1708 - 1717.

[4] Hofkens, J.; Vosch, T.; Maus, M.; Köhn, F.; Cotlet, M.; Weil, T.; Herrmann, A.; Müllen, K.; de Schryver, F. C. Conformational Rearrangements in and Twisting of a Single Molecule. Chem. Phys. Lett. 2001, 333, 255-263.

[5] Vallée, R. A. L.; Tomczak, N.; Kuipers, L.; Vancso, G. J.; van Hulst, N. F.; Single Molecule Lifetime Fluctuations Reveal Segmental Dynamics in Polymers. Phys. Rev. Lett. 2003, 91, 38301.

[6] Duke, C.B.; Fabish, T.J.; Charged-Induced Relaxation in Polymers, Phys. Rev. Lett., 1976, 37, $1075-1078$.

[7] Duke, C.B.; Fabish, T.J.; Molecular Carge States and Contact Charge Exchange in Polymers. J. Appl. Phys., 1977, 48, $4256-4266$.

[8] Hippius, C.; Multichromophoric Arrays of Perylene Bisimide Dyes - Synthesis and Optical Properties (Doctoral Dissertation), University of Würzburg, 2007.

[9] Sbalzarini, I. F.; Koumoutsakos, P.; Feature Point Tracking and Trajectory Analysis for Video Imaging in Cell Biology. J. Struct. Biol., 2005, 151, 182 - 1958. 